\title{
Smoking, alcohol and drug use in youth and adults with attention-deficit hyperactivity disorder
}

\author{
Sydney Osland, Lauren Hirsch and Tamara Pringsheim
}

\section{Background}

Previous research suggests a relationship between attentiondeficit hyperactivity disorder (ADHD) and smoking, alcohol and illicit drug use, however most studies have focused on adolescents or young adults, or clinically ascertained samples.

\section{Aims}

To analyse population-based data on the relationship between ADHD and at-risk health behaviours in adolescents and adults.

\section{Method}

Data were derived from a Statistics Canada population-based health survey. The association between the diagnosis of ADHD and smoking, alcohol use, and illicit drug use was examined.

\section{Results}

Individuals with ADHD started smoking at a younger age. They consumed more alcoholic drinks on drinking days, and women with ADHD were more likely to engage in binge drinking. Women over the age of 25 and men with ADHD were more likely to meet alcohol-dependence lifetime criteria. People with ADHD were at a greater risk of drug misuse and dependence.

\section{Conclusions}

People with ADHD are more likely to partake in at-risk behaviours.

\section{Declaration of interest}

None.

\section{Copyright and usage}

(C) The Royal College of Psychiatrists 2017. This is an open access article distributed under the terms of the Creative Commons Non-Commercial, No Derivatives (CC BY-NC-ND) license.
Attention-deficit hyperactivity disorder (ADHD) is an early-onset neurodevelopmental disorder. Originally believed to only be present in children, studies have indicated that there are numerous cases in which ADHD persists into adulthood. ${ }^{1}$ One of the first and most representative epidemiological studies performed to examine adult ADHD found that approximately $4.4 \%$ of Americans met diagnostic criteria presented in DSM-IV. ${ }^{2}$ Comorbidities are common in people with ADHD, with over half having at least one comorbid condition, the most prevalent being conduct disorder and oppositional defiant disorder. ${ }^{3}$

Impulsivity is one of the core features of ADHD. ${ }^{4}$ Evidence showing that people with ADHD have lower levels of neurotransmitters, such as dopamine, has led to the speculation that individuals with ADHD participate in risky behaviors to stimulate dopamine release. ${ }^{5}$ As such, studies focusing on the nature of the relationship between ADHD and risk-taking behaviours are essential to improve our understanding of this condition and treatment needs. One of the more highly studied health behaviours in people with ADHD is tobacco smoking. It has been suggested that ADHD increases the likelihood of smoking in childhood and adolescence, lowers the overall age at first use, and increases the number of cigarettes smoked, and this pattern continues into adulthood. ${ }^{6-8}$ Charach et al ${ }^{9}$ previously completed a meta-analysis of alcohol and substance misuse and childhood ADHD as a risk factor for these disorders. They concluded that alcohol misuse occurred more frequently in people who had received a childhood diagnosis of ADHD, compared with those who had not. People with ADHD were possibly at a greater risk of cannabis misuse and psychoactive substance use disorders, and were at a significantly greater risk of other non-alcohol drug use disorders, but the degree of risk could not be determined. Recently, Brinkman et al ${ }^{10}$ also found that the age at initiation of alcohol consumption was significantly lower in adolescents with ADHD compared with healthy individuals.

This study seeks to extend the literature on ADHD and at-risk health behaviours in adults and adolescents using Canadian population-based data to present a more comprehensive understanding of how this neurodevelopmental disorder can be associated with behaviours that affect physical and mental health in the community. Although previous research has demonstrated a relationship between ADHD and smoking, alcohol and drug use, studies using Canadian data are few in number, have focused primarily on adolescents or young adults, or have used clinically ascertained samples. Additionally, some results have been contradictory or inconclusive and we aim to add clarity to this issue. Although a recent Canadian study using a population-based sample found that ADHD symptoms are related to use of alcohol and cannabis, the study was comprised only of Ontario residents and therefore may not be representative of the entire Canadian population. ${ }^{11}$ Additionally, although they did examine cannabis use, they did not examine other illicit drug use. This study is therefore necessary to add the limited Canadian literature on ADHD and risky health behaviours. We hypothesised that the diagnosis of ADHD would be associated with higher odds of smoking, alcohol and drug use behaviours and related impairment.

\section{Method}

This study was approved by the Social Sciences and Humanities Research Council of Canada.

\section{Participants}

The data used were obtained from the Canadian Community Health Survey - Mental Health (CCHS-Mental Health) 2012 (released 18 September 2013). The CCHS-Mental Health is a cross-sectional survey designed to collect information on health status, healthcare utilisation and health determinants for the Canadian population. The survey covers the population aged 15 and over, living in the 10 provinces for a total sample size of 25 113. Desired sample size for the CCHS-Mental Health was 27500 based on what was required for reliable provincial-level estimates and the budget available. To select respondents, geographical clusters were first selected, followed by household selection within each cluster and finally the random selection of one 
respondent per household. Persons living on reserves and other Aboriginal settlements, full-time members of the Canadian Forces and the institutionalised population were excluded from the survey. These exclusions account for approximately 3\% of the Canadian population who are aged 15 and above. The combined household and person response rate was $68.9 \%$.

\section{Survey design}

The CCHS-Mental Health was developed by Statistics Canada in association with stakeholders from Health Canada, the Public Health Agency of Canada, the Provincial Health Ministries and an advisory committee consisting of specialists and academic experts. This survey also utilises computer-assisted interviewing. The CCHS has a common content section collected from all respondents, and an optional content component specific to the data needs of the individual provinces and territories, and a rapid response content component which is offered to organisations interested in national estimates based on emerging or specific issues related to population health. Our study utilises this survey for data specific to demographics (education, income), smoking (smoking status, cigarettes per day, age at first cigarette smoking), alcohol use (drinks per day, binge drinking, alcohol dependence) and illicit drug use (use of various drugs including prescription drugs for non-medicinal purposes).

\section{Diagnosis of ADHD}

CCHS-Mental Health survey participants were asked about a diagnosis of ADHD. The CCHS-Mental Health survey stated 'Remember, we're interested in conditions diagnosed by a health professional and are expected to last or have already lasted 6 months or more'. Following this it was asked, 'Do you have attention-deficit disorder'? to which the respondents answered either yes or no. The survey did not specify whether the diagnosis was attention-deficit disorder (ADD) or ADHD. Respondents who answered 'yes' were placed in the ADHD group, to be compared with those who had answered 'no', the control group, in terms of smoking, alcohol and illicit drug use.

\section{Variables studied}

We examined variables related to smoking, alcohol use and illicit drug use. We also examined demographic features of the populations including age, gender, household income, level of education achieved and employment. Additionally, we compared prescribed stimulant use in those with ADHD compared with those without. With respect to smoking, we looked at the age at which the current or past daily smokers started smoking daily, the age respondents had smoked their first whole cigarette, whether they were a current smoker, whether they had ever smoked and the number of cigarettes smoked per day for both current daily and current occasional smokers. Alcohol use variables examined included the mean number of drinks per day, whether the respondent had had binge-drinking episodes ( 5 or more drinks in 1 day) within the past year, whether they had alcohol dependence issues at a point in their life, and whether they had ever sought professional help when they had alcohol-related issues. With respect to illicit drug use, we examined whether respondents have ever met the criteria for marijuana misuse, whether they have ever met the criteria for marijuana dependence, whether they met the criteria for drug misuse within their lifetime (excluding cannabis) and whether they met the criteria for drug dependence (excluding cannabis) within their lifetime. In total, the CCHS-Mental Health survey has 164 questions pertaining to smoking, alcohol and drug use. Derived variables were used to examine alcohol dependence and substance misuse and dependence. To meet the alcohol-dependence lifetime criteria, respondents had to report at least three symptoms related to tolerance, withdrawal, increased consumption, attempts to quit, time lost, reduced activities and continued drinking, and three or more of these symptoms must have occurred during the same 12 -month period. The same criteria were required to meet marijuana lifetime dependence and drug (excluding cannabis) lifetime dependence. Additionally, to meet marijuana misuse lifetime criteria, respondents must not have experienced dependence and must meet one of the four criteria for misuse, which include recurrent use leading to a failure to complete obligations, use in situations when it is physically hazardous, related legal problems and continued use regardless of social and interpersonal problems caused or worsened by use. ${ }^{12}$ Criteria are the same for drug (excluding cannabis) lifetime misuse. Variables with dichotomous outcomes are presented as proportions, and continuous variables are presented as means, both with associated 95\% confidence intervals (CIs).

\section{Statistical analysis}

All analyses were completed at the Prairie Regional Data Center using STATA Data Analysis and Statistical Software, version $14{ }^{13}$ Weighted and bootstrapped estimates were calculated for categorical variables as proportions to represent the prevalence, as well as 95\% CI to assess for statistical significance. Sampling and bootstrap weights were applied for all estimates reported by the CCHS. These weights were applied to account for over or under sampling in the survey, and to adjust the variance through resampling so that it more accurately represents the entire Canadian population, respectively. Logistic regression was used to calculate odds ratios and 95\% CI to describe the association between ADHD and smoking, alcohol and drug use. Effect measure modification by age or gender was evaluated with the Wald test to assess the significance of crossproduct interaction terms. The presence of confounding was assessed by examining changes in the beta estimate following backward elimination of the interaction terms. Estimated odds ratios presented here have been adjusted for both age and gender. When an interaction between age and gender was significant (using a priori significance level of 0.05 ), respondents were stratified by age (age 15-25 compared with those age 26 and over) and gender (female or male) and odds ratios are presented for each of the four groups (female, age 25 and under; female, age over 25; male, age 25 and under; and male, age over 25). The decision to stratify into these age categories was based on indications that adolescence, and the risk-taking behaviour that is associated with adolescence, may last until age 25 and not the previously suggested age of $18 .{ }^{14}$ Additionally, many people age 25 and under are around peers who may encourage risky behaviours. Also, because of differences in socioeconomic status (SES) between the ADHD and control groups, we adjusted for income when possible. The relationship between SES and smoking is well established, with lower SES being correlated with higher incidence of smoking. ${ }^{15}$ The relationship between SES and alcohol and substance use is less established however, with some studies showing a positive correlation, others showing a negative correlation and others showing no relationship. ${ }^{16-19}$ Thus, although we did determine odds ratios adjusted for income differences between groups, these adjustments may not be necessary or reflective of the relationship between SES and alcohol or substance use.

\section{Results}

\section{Demographics}

A total of 24424 respondents answered 'no' to having received a diagnosis of ADD or ADHD, whereas 670 respondents answered 'yes', with an estimated prevalence of $2.63 \%$ (95\% CI 2.32-2.94). The ADHD population consisted of a significantly younger 
population, (mean 33.96 years; 95\% CI 32.38-35.54) than the control population (mean 45.96 years; 95\% CI 45.83-46.10). Additionally, the ADHD group included significantly more males $(62.13 \%$ male; $95 \%$ CI 56.97-67.40) than the control group (48.92\% male; $95 \%$ CI $48.77-49.07$ ). A total of $31.46 \%$ (95\% CI 26.28-36.64) of individuals with ADHD had ever used a prescribed stimulant, compared with $1.63 \%$ (95\% CI 1.42-1.84) of the control population. Owing to the differences in age between the ADHD and control populations, the remainder of the demographic variables that were felt to be highly influenced by age were compared between the ADHD and control populations in those aged 25 and under, and those over 25 (Table 1). Even after controlling for age, differences between the ADHD and control populations persisted for household income for both age groups, and employment only for those aged 25 and under.

\section{Smoking}

Results are summarised in Table 2. The age that respondents with ADHD smoked their first whole cigarette was significantly lower than those without, as was the age that they started smoking daily (if they had ever been or currently were a daily smoker). A higher proportion of people with ADHD were current smokers compared with the control group and had ever smoked compared with the control group. For current daily smokers, there was no significant difference between mean cigarettes smoked per day. Additionally, there was no significant difference in mean cigarettes per day for occasional smokers. Results presented in Table 2 are adjusted for age and gender, but because of the potential of SES to act as a confounder in the relationship between ADHD and smoking, we also examined odds ratios including this additional income adjustment, presented in Table 3. After adjusting for income, there is no association between ADHD and having ever smoked or being a current smoker.

\section{Alcohol use}

Results are summarised in Table 4. Individuals with ADHD reported a significantly higher number of alcoholic drinks per day on drinking days in the past 12 months. With respect to episodes of binge drinking ( 5 or more drinks in 1 day) for people who had drank in the past 12 months, a significantly higher proportion of people with ADHD had a binge-drinking episode more than once a month compared with the control population. Women with ADHD aged over 25 had 4.36 (95\% CI 1.86-10.23) times the odds of having more than one binge-drinking episode in the past 12 months. Women aged 25 and under with ADHD had 2.41 (95\% CI 1.18-4.92) times the odds of having more than one episode. Neither age group of men was significantly more likely to have a binge-drinking episode than controls. A significantly higher proportion of people with ADHD met the alcohol dependence lifetime criteria (have had dependence issues for at least one 12 month period during their lifetime) compared with the control population. Women with ADHD aged over 25 had higher odds of meeting the alcohol dependence criteria compared with those without, whereas women under 25 years did not. Men with ADHD of age under and over 25 had higher odds of meeting the alcohol dependence lifetime criteria than controls. Finally, a significantly higher proportion of people with ADHD who had alcohol-related issues had ever sought any form of professional help compared with the control population who had experienced issues with drinking and had 2.63 (95\% CI 1.54-4.49) times the odds of seeking professional help. Odds ratios adjusted for age, gender and income are presented in Table 3, which illustrate that after adjusting for low SES, the relationship between ADHD and alcohol use is unchanged.

\section{Substance use}

Results are summarised in Table 5. People with ADHD had higher odds of meeting the criteria for marijuana misuse within their lifetime compared with controls. Additionally, a significantly higher proportion of people with ADHD met the criteria for marijuana dependence within their lifetime, with an odds ratio of 3.41 (95\% CI 2.00-5.80). For drug misuse (excluding cannabis and including sedatives, stimulants, analgesics, cocaine, club drugs, hallucinogens, heroin, inhalants and any other illegal drugs) within their lifetime, those with ADHD had significantly increased odds of meeting the lifetime misuse criteria compared with controls. A significantly higher proportion of people with ADHD met the drug- (excluding cannabis) dependence lifetime criteria compared with the control population, with an odds ratio of 5.74 (95\% CI 3.62-9.09). Odds ratios adjusted for age, gender and income are presented in Table 3. After adjusting for SES, the association between ADHD and marijuana and drug misuse and marijuana dependence was no longer statistically significant, largely because of widening of the confidence intervals around these estimates.

\section{Discussion}

The estimated prevalence of ADHD determined in this study of $2.63 \%$ (95\% CI 2.32-2.94) is consistent with previous Canadian studies placing the prevalence at around 3\% (3.30\%, 95\% CI 2.75-3.85). ${ }^{20}$ Demographics and some socioeconomic factors between the ADHD and control groups differed significantly. The ADHD group is made up of a younger and more male demographic. Examination of sociodemographic factors indicated that people

\begin{tabular}{|c|c|c|c|c|}
\hline \multirow[b]{2}{*}{ Variable } & \multicolumn{2}{|c|}{25 years and under } & \multicolumn{2}{|c|}{ Over 25 years } \\
\hline & Controls & ADHD population & Controls & ADHD H \\
\hline Household incomes under $\$ 30000$, \% & $18.46(16.49-20.43)$ & $28.64(21.36-35.93)$ & $16.33(15.49-17.16)$ & $27.48(21.67-33.28)$ \\
\hline Completed any post-secondary school, \% & $49.41(46.93-51.88)$ & $38.76(30.05-47.48)$ & $69.94(68.77-71.10)$ & $63.23(56.03-70.43)$ \\
\hline Employed, \% & $61.09(58.77-63.41)$ & $50.55(42.63-58.47)$ & $70.38(69.41-71.35)$ & $67.20(60.62-73.79)$ \\
\hline
\end{tabular}

\begin{tabular}{|c|c|c|c|}
\hline Variable & Controls & ADHD population & Odds ratio $(95 \% \mathrm{Cl})$ \\
\hline Mean age smoked first whole cigarette, years & $16.17(16.05-16.29)$ & $14.89(14.29-15.49)$ & \\
\hline Mean age started smoking daily (if a current or past daily smoker), years & $17.97(17.82-18.11)$ & $16.85(16.19-17.52)$ & \\
\hline Current smoker (daily or occasional), \% & $20.59(19.69-21.48)$ & $35.42(29.84-41.00)$ & $1.79(1.37-2.32)$ \\
\hline Have ever smoked, \% & $59.75(58.68-60.81)$ & $64.63(58.42-70.84)$ & $1.51(1.14-2.00)$ \\
\hline Mean cigarettes per day (current daily smokers) & $14.81(14.39-15.23)$ & $15.42(13.83-17.01)$ & \\
\hline Mean cigarettes per day (current occasional smokers) & $3.18(2.90-3.46)$ & $5.33(3.42-7.25)$ & \\
\hline
\end{tabular}




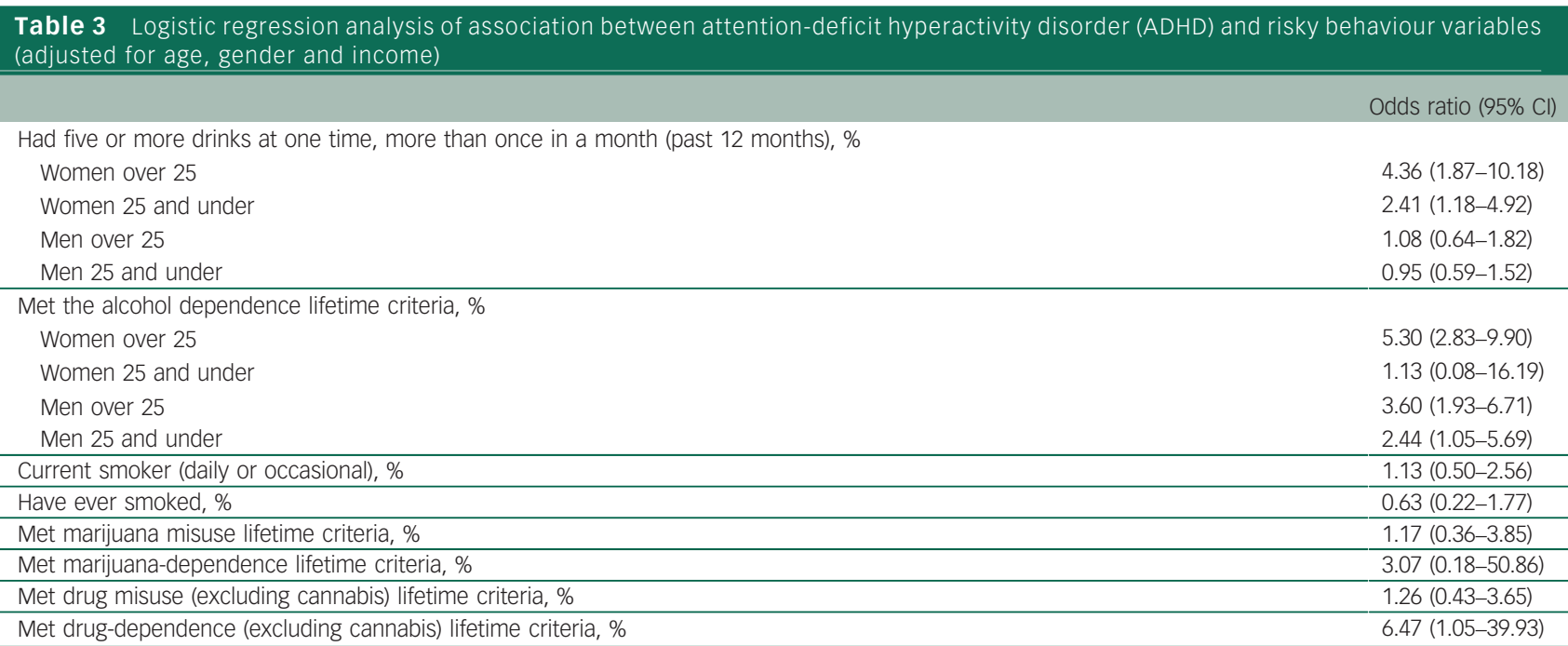

\begin{tabular}{|c|c|c|c|}
\hline Variable & Controls & ADHD & Odds ratio $(95 \% \mathrm{Cl})$ \\
\hline Mean number of drinks per day (on days drinking in past 12 months), \% & $2.65(2.59-2.71)$ & $3.67(3.23-4.10)$ & \\
\hline Had five or more drinks at one time, more than once in a month (past 12 months), \% & $17.32(16.43-18.21)$ & $27.47(21.37-33.56)$ & \\
\hline Women over 25 & & & $4.36(1.86-10.23)$ \\
\hline Women 25 and under & & & $2.41(1.18-4.92)$ \\
\hline Men over 25 & & & $1.08(0.62-1.82)$ \\
\hline Men 25 and under & & & $0.95(0.59-1.51)$ \\
\hline Met the alcohol-dependence lifetime criteria, \% & $2.83(2.52-3.13)$ & $9.79(6.65-12.92)$ & \\
\hline Women over 25 & & & $6.17(3.32-11.46)$ \\
\hline Women 25 and under & & & $1.19(0.08-16.85)$ \\
\hline Men over 25 & & & $3.74(2.02-6.91)$ \\
\hline Men 25 and under & & & $2.66(1.14-6.20)$ \\
\hline Have ever sought professional help for alcohol-related issues, \% & $11.08(9.86-12.30)$ & $20.64(12.27-29.00)$ & $2.63(1.54-4.49)$ \\
\hline
\end{tabular}

$\begin{aligned} & \text { Table } \mathbf{5} \text { Proportions and logistic regression analysis of association between attention-deficit hyperactivity disorder (ADHD) and } \\
& \text { substance use variables (adjusted for age and gender or stratified when age or gender interaction present) }\end{aligned}$
\begin{tabular}{|cccc|}
\hline & Controls & ADHD population & Odds ratio (95\% Cl) \\
\hline Met marijuana misuse lifetime, \% & $4.98(4.52-5.45)$ & $13.67(10.16-17.19)$ & $2.14(1.55-2.96)$ \\
\hline Met marijuana-dependence lifetime criteria, \% & $1.42(1.17-1.69)$ & $7.18(4.06-10.29)$ & $3.41(2.00-5.80)$ \\
\hline Met drug misuse (excluding cannabis) lifetime criteria, \% & $1.96(1.68-2.23)$ & $6.88(4.39-9.37)$ & $3.19(2.14-4.77)$ \\
\hline Met drug-dependence (excluding cannabis) lifetime criteria, \% & $1.65(1.39-1.91)$ & $10.91(6.78-15.02)$ & $5.74(3.62-9.09)$ \\
\hline
\end{tabular}

with ADHD were living in lower income households, and those with ADHD age 25 and under were less likely to be employed. Previous research has suggested that people with ADHD receive significantly fewer years of education compared with their non-affected counterparts specifically with respect to completing college and some postgraduate work, but findings on the differences in proportion of people with and without ADHD who finished high school, some college or received a postgraduate degree, have been inconclusive. $^{21,22}$ The deficits seen in academic performance could be predicted to translate into employment issues such as missing more work, troubles finding and sticking with a job and lower income. Previous studies have also found that a lower percentage of people with ADHD are employed, and earn an income significantly lower than the control population. ${ }^{22}$

People with ADHD were at a significantly greater risk of being current daily or occasional smokers as well as having ever smoked, but this relationship did not persist when adjusting for SES differences between the ADHD and control populations. Individuals with ADHD smoked their first whole cigarette and started smoking daily at a significantly younger age than the control group. There was no significant difference in mean number of cigarettes smoked between groups. Prior studies have found that people with $\mathrm{ADHD}$ started smoking at a lower age and were more likely to be smokers or have smoked previously. ${ }^{6-8}$ These studies on smoking and ADHD adjusted for SES by eliminating the entire lowest socioeconomic class from the study, ${ }^{7}$ adjusted through data on parental education and receipt of public assistance ${ }^{8}$ or did not adjust for SES and used other population data available at the time as their control group. ${ }^{6}$ When controlling only for age and gender, our results were in keeping with previous studies on the association between ADHD and smoking. After adjusting for SES, however, our 95\% CI crossed zero, suggesting a relationship between ADHD and smoking may or may not be present. This could be because of the complex and bidirectional association between ADHD and low $\mathrm{SES}$, as well as smoking. Individuals with ADHD are more likely to have low SES which may be partly because of previous mentioned differences noted in education and employment. Low SES of parents is a risk factor for ADHD in children, ${ }^{23}$ and individuals with low SES are more likely to smoke, ${ }^{15}$ a known risk factor for the development of ADHD in offspring. ${ }^{24}$ ADHD is a highly heritable disorder, and it is not possible to determine the relative contribution of genetic $v$. environmental risk factors in affected 
individuals. The weakening of the association between ADHD and smoking when adjusting for SES may be because of effect mediation, confounding or both.

A significantly higher proportion of people with ADHD have had more than one binge-drinking episode a month compared with the control population. Women aged over 25 were at a significantly higher risk, as well as women 25 and under, with no significant difference in risk in men. The lack of a significant difference found in males with ADHD compared with those without may be related to findings that binge drinking in men is significantly more common than in women, with studies suggesting that men are three times as likely to binge drink. ${ }^{25}$ Our alcohol use findings are consistent with previous literature, which have indicated that people with ADHD are much more likely to develop alcohol use disorders in adulthood, ${ }^{9-10}$ and we have shown that this relationship persists after adjusting for income.

Respondents with ADHD were also found to be at a greater risk of participating in substance use behaviours. They were significantly more at risk of having marijuana-related misuse and dependence issues, as well as misuse and dependence of other drugs not including marijuana (e.g. club drugs, cocaine). Although the variables examined were slightly different between studies, overall our results confirm and extend the findings of Charach and colleagues' meta-analysis which indicated a potential increased risk in cannabis use disorders and psychoactive drug use disorders, and a significant but undetermined degree of risk for other non-alcohol drug use disorders. ${ }^{9}$ After adding an adjustment for SES, confidence intervals around our estimates widened, resulting in point estimates that suggest a higher odds, but confidence intervals which cross the null value. Overall, we found increased risk for substance use and dependence disorders, for all types of drugs, in people with ADHD.

The findings of this population-based study confirm that people with ADHD are more likely to participate in at-risk health behaviours, specifically alcohol and substance use, but the relationship between ADHD and smoking behaviours is less clear. Although previous studies examining the association between smoking and ADHD found that the relationship persisted after adjusting for SES, ${ }^{7,8}$ our results suggests otherwise. This may be because of differences between studies in the variables used to define and account for SES. Previous studies used methods such as eliminating the entire lowest SES class from their analysis, or made adjustments for SES based on education levels of parents and social service utilisation, rather than the respondents' education level or income level directly. Thus, our study demonstrates a need to further examine the role of SES in the relationship between ADHD and smoking using a standard approach to define and account for SES.

Potential reasons for the associations between ADHD and smoking, alcohol and substance use include problems with impulse control, or a possible desire to self-treat ADHD symptoms because of inadequate medical or psychosocial support. Impulsivity is increased in $\mathrm{ADHD}$, making this a viable explanation for the risktaking behaviours seen, but there is evidence supporting the selfmedication theory for increased smoking, drinking and substance use as well. A controlled family study examining self-medication found that $36 \%$ of people with ADHD smoked and used substances to self-medicate (33\% for mood changing and 3\% to aid sleep), whereas $25 \%$ used to get high and the remaining $39 \%$ had other or unknown motivations. ${ }^{26}$ It has been speculated that tobacco smoking is used as a method of self-medication because of its enhancing effect on behavioural inhibition abilities and cognitive functioning. ${ }^{27}$ Additional studies have suggested that substances such as cannabis and cocaine lead to neurotransmitter release, specifically dopamine, which would therefore reduce the main symptoms of ADHD, supporting the self-medication hypothesis of substance use. ${ }^{28}$ Therefore, impulsivity and self-medication may both be factors contributing to the significant increases in risktaking behaviours seen in the ADHD population. This raises the question as to whether people with $\mathrm{ADHD}$ are receiving proper treatment to manage symptoms, and if not, what types of alternative treatments may be valuable. A balanced diet with regular exercise, cognitive-behavioural therapy and pharmacological treatments have all been suggested to manage the risk-taking behaviours that people with ADHD partake in. ${ }^{29}$ As we could not examine what treatments respondents had received, and whether there was a relationship between type of treatment, symptom severity and risk taking behaviours, we cannot indicate what treatment methods may be the most effective. This is an area for future examination and an important step in addressing how to reduce the increased amount of risk-taking behaviours that people with ADHD partake in. Additionally, children aged under 15 may be included in future studies by adding variables more specific to risk-taking behaviours that younger children may participate in (i.e. playground play decisions leading to injury) as there is evidence that ADHD leads to an increased risk of injury. ${ }^{30}$ In general, future study is needed to determine additional factors influencing the findings obtained in this examination, and others of a similar nature.

A limitation in this study was our inability to stratify completely by age and gender, given that the two groups were significantly different demographically. However, measures were taken to adjust odds ratios for age and gender, as well as stratify into two age groups (age 25 and under, and over 25) and by gender when an interaction between age and gender was determined. Additionally, participants were asked whether they had ever received a diagnosis of ADD and not ADHD specifically. This relied on self-reporting and did not indicate diagnostic criteria used. Thus, the ADHD population we examined may include people who no longer had symptoms or were incorrectly diagnosed, and the control population may contain people with the disorder who were never diagnosed. This underdiagnosis may be a critical issue in more remote survey areas with less access to mental health physicians. The inclusion of undiagnosed individuals with ADHD in the control group however would only minimise any difference found in outcomes between groups, many of which were statistically significant. The CCHS relies on the self-report of health-professional diagnosed conditions for its determination of whether an individual has ADHD, as well as all other medical conditions evaluated in their survey. Validation studies for the self-report of diagnosed ADHD have not been performed to our knowledge, however validation of self-reported chronic conditions has been done for a number of other psychiatric and neurological disorders. ${ }^{31-34}$ For example, a validation study of self-reported diagnosis of depression found that $74.2 \%$ of individuals self-reporting a physician diagnosis of depression were classified as depressed using the Structured Clinical Interview for DSM-IV (SCID-I). ${ }^{35}$ Although misclassification bias is possible with the use of self-reported diagnoses, we believe there is adequate data from other conditions to support the use and validity of this method.

The importance of this examination is that it is one of the first Canadian studies using population-based data to ascertain differences in risk-taking behaviours between people with and without ADHD. Our findings indicate that there is a significantly greater risk that people who have been diagnosed with ADHD will participate in alcohol use and substance use. These findings are important as they confirm previous findings from studies using clinically ascertained samples. However, our analysis of smoking behaviours suggests that the increased risk of smoking in this group is because of the association between ADHD and SES, a known risk factor for smoking. Additionally, previous literature 
has focused almost exclusively on the relationship between ADHD and risk-taking behaviours in adolescents and young adults. Thus, it is unclear whether findings are because of the combination of the impulsivity that accompanies ADHD and a predisposition for younger people to act in a riskier way, and not just the presence of $\mathrm{ADHD}$ alone. As ADHD is no longer considered a disorder that only affects children or adolescents, extending the current literature past young adults is crucial. Our study determined that people over the age of 25 were also at greater risk of alcohol and substance misuse-related behaviours. This provides us with insight that this is not just an issue concerning adolescents and young adults, a conclusion which could not be drawn from previous literature available. Owing to the compromised health that may result from behaviours examined in this study, it is essential that current treatment methods address the complex health needs of individuals with $\mathrm{ADHD}$, and that these treatments do not cease once adulthood is reached.

Sydney Osland, BSC, Cumming School of Medicine, University of Calgary, Calgary, Alberta, Canada; Mathison, Centre for Mental Health Research and Education, Calgary, Alberta, Canada; Lauren Hirsch, MSc, Cumming School of Medicine, University of Calgary, Calgary, Alberta, Canada; Tamara Pringsheim, MD, Cumming School of Medicine, University of Calgary, Calgary, Alberta, Canada; Department of Clinical Neurosciences, Psychiatry, Pediatrics and Community Health Sciences, Mathison Centre for Mental Health Research and Education, Calgary, Alberta, Canada

Correspondence: Tamara Pringsheim, Mathison Centre for Mental Health Research and Education, TRW Building, 3280 Hospital Drive NW, T2N 4Z6 Calgary, AB, Canada. Email: tmprings@ucalgary.ca

First received 10 Nov 2016, final revision 31 Mar 2017, accepted 10 Apr 2017

\section{References}

1 Faraone SV, Biederman J, Mick E. The age-dependent decline of attention deficit hyperactivity disorder: a meta-analysis of follow-up studies. Psychol Med 2006; 36: 159-65.

2 Kessler RC, Adler L, Barkley R, Biederman J, Conners CK, Demler O, et al. The prevalence and correlates of adult ADHD in the United States: results from the national comorbidity survey replication. Am J Psychiatry 2006; 163: 716-23.

3 Spencer TJ, Biederman J, Mick E. Attention-deficit/hyperactivity disorder: diag nosis, lifespan, comorbidities, and neurobiology. Ambul Pediatr 2007; 7(1 suppl): 73-81.

4 Pappas D. ADHD Rating Scale-IV: checklists, norms, and clinical interpretation. J Psychoeduc Assess 2006; 24: 172-8

5 Blum K, Chen AL, Braverman ER, Comings DE, Chen TJ, Arcuri V, et al. Attentiondeficit-hyperactivity disorder and reward deficiency syndrome. Neuropsychiatr Dis Treat 2008; 4: 893-918.

6 Pomerleau OF, Downey KK, Stelson FW, Pomerleau CS. Cigarette smoking in adult patients diagnosed with attention deficit hyperactivity disorder. J Subst Abuse 1995; 7: 373-8.

7 Milberger S, Biederman J, Faraone SV, Chen L, Jones J. ADHD is associated with early initiation of cigarette smoking in children and adolescents. J Am Acad Child Adolesc Psychiatry 1997; 36: 37-44

8 Kollins SH, Mcclernon FJ, Fuemmeler BF. Association between smoking and attention-deficit/hyperactivity disorder symptoms in a population-based sample of young adults. Arch Gen Psychiatry 2005; 62: 1142-7.

9 Charach A, Yeung E, Climans T, Lillie E. Childhood attention-deficit/hyperactivity disorder and future substance use disorders: comparative meta-analyses. J Am Acad Child Adolesc Psychiatry 2011; 50: 9-21.

10 Brinkman WB, Epstein JN, Auinger P, Tamm L, Froehlich TE. Association of attention-deficit/hyperactivity disorder and conduct disorder with early tobacco and alcohol use. Drug Alcohol Depend 2015; 147: 183-9.

11 Kolla NJ, van der Maas M, Toplak ME, Erickson PG, Mann RE, Seeley J, et al. Adult attention deficit hyperactivity disorder symptom profiles and concurrent problems with alcohol and cannabis: sex differences in a representative, population survey. BMC PSychiatry 2016; 16: 50

12 American Psychiatric Association. Diagnostic and Statistical Manual of Mental Disorders, Fourth Edition, Text Revision (DSM-IV-TR). APA, 2000
13 StataCorp. Stata Statistical Software: Release 14. StataCorp LP, 2015

14 Arnett JJ. Emerging adulthood: a theory of development from the late teens through the twenties. Am Psychol 2000; 55: 469-80.

15 Hiscock R, Bauld L, Amos A, Fidler JA, Munafo M. Socioeconomic status and smoking: a review. Ann NY Acad Sci 2012; 1248: 107-23.

16 Hanson MD, Chen E. Socioeconomic status and health behaviours in adolescence: a review of the literature. J Behav Med 2007; 30: 263-85.

17 Patrick ME, Wightman P, Schoeni RF, Schulenberg JE. Socioeconomic status and substance use among young adults: a comparison across constructs and drugs. J Stud Alcohol Drugs 2012; 73: 772-82.

18 Bonevski B, Regan T, Paul C, Baker AL, Bisguera A. Associations between alcohol, smoking, socioeconomic status and comorbidities: evidence from the 45 and up study. Drug Alcohol Rev 2014; 33: 169-76.

19 Charitonidi E, Studer J, Gaume J, Gmel G, Daeppen JB, Bertholet N. Socioeconomic status and substance use among Swiss young men: a population-based crosssectional study. BMC Public Health 2016; 16: 333.

20 Vingilis E, Erickson PG, Toplak ME, Kolla NJ, Mann EM, Seeley J, et al. Attention deficit hyperactivity disorder symptoms, comorbidities, substance use, and social outcomes among men and women in a Canadian sample. Biomed Res Int 2015; 2015: 1-8.

21 Murphy KR, Barkley RA, Bush T. Young adults with attention deficit hyperactivity disorder: subtype differences in comorbidity, educational, and clinical history. J Nerv Ment Dis 2002; 190: 147-57.

22 Biederman J, Faraone SV. The effects of attention-deficit/hyperactivity disorder on employment and household income. MedGenMed 2006; 8: 12.

23 Russell $A E$, Ford $T$, Russell G. Socioeconomic associations with ADHD: findings from a mediation analysis. PLOS One 2016; 10: 1-16.

24 Langley $\mathrm{K}$, Rice $\mathrm{F}$, van den Bree MB, Thapar A. Maternal smoking during pregnancy as an environmental risk factor for attention deficit hyperactivity disorder behavior. A review. Minerva Pediatr 2005; 57: 359-71.

25 Naimi TS, Brewer RD, Mokdad A, Denny C, Serdula MK, Marks JS. Binge drinking among US adults. JAMA 2003; 289: 70-5.

26 Wilens TE, Adamson J, Sgambati S, Whitley J, Santry A, Monuteaux MC, et al. Do individuals with ADHD self-medicate with cigarettes and substances of abuse? Results from a controlled family study of ADHD. Am J Addict 2007; 16 (suppl 1): 14-21; quiz 22-3.

27 Gray KM, Upadhyaya HP. Tobacco smoking in individuals with attention-deficit hyperactivity disorder: epidemiology and pharmacological approaches to cessation. CNS Drugs 2009; 23: 661-8.

28 Silva $N$, Szobot $C M$, Shih $M C$, Hoexter MQ Anselmi CE, Pechansky $F$, et al. Searching for a neurobiological basis for self-medication theory in ADHD comorbid with substance use disorders: an in vivo study of dopamine transporters using (99m)TC-TRODAT-1 SPECT. Clin Nucl Med 2014; 39: e129-34.

29 Mall M, King-Hill S, Holland P. Managing risk-taking behaviour in children and young people with ADHD. Br J School Nurs 2013; 8: 483-7.

30 Schwebel DC, Roth DL, Elliott MN, Visser SN, Toomey SL, Shipp EM, et al. Association of externalizing behavior disorder symptoms and injury among fifth graders. Acad Pediatr 2011; 11: 427-31.

31 Haapanen $\mathrm{N}$, Miilunpalo $\mathrm{S}$, Pasanen $\mathrm{M}$, Oja $\mathrm{P}$, Vuori I. Agreement between questionnaire data and medical records of chronic diseases in middle-aged and elderly Finnish men and women. Am J Epidemiol 1997; 145: 762-9.

32 Martin LM, Leff M, Calonge N, Garrett C, Nelson DE. Validation of self-reported chronic conditions and health services in a managed care population. Am J Prev Med 2000; 18: 215-8.

33 Okura Y, Urban LH, Mahoney DW, Jacobsen SJ, Rodeheffer RJ. Agreement between self-report questionnaires and medical record data was substantial for diabetes, hypertension, myocardial infarction and stroke but not for heart failure. J Clin Epidemiol 2004; 57: 1096-103.

34 Brooks DR, Avetisyan R, Jarrett KM, Hanchate A, Shapiro GD, Pugh MJ, et al. Validation of self-reported epilepsy for purposes of community surveillance. Epilepsy Behav 2012; 23: 57-63.

35 Sanchez-Villegas A, Schlatter J, Ortuno F, Lahortiga F, Pla J, Benito S, et al. Validity of a self-reported diagnosis of depression among participants in a cohort study using the structured clinical interview for DSM-IV (SCID-I). BMC PSychiatry 2008; 8: 43 